\title{
Graphical Investigation of Threshold Choice Effect on Odds Ratio Related to Prognostic Factors in Stroke Recovery
}

\author{
Marco Iosa*, Giovanni Morone, Augusto Fusco and Stefano Paolucci
}

\author{
Clinical Laboratory of Experimental Neurorehabilitation, I.R.C.C.S. Fondazione Santa Lucia, via Ardeatina \\ 306, 00179, Rome, Italy
}

\begin{abstract}
The aim of this study was to assess the effects of the arbitrary choices of threshold-values for dichotomizing not binary factors on the computation of odds ratio (OR) for the identification of prognostic factors, in particular of motor recovery after stroke.

Data of a sample of 1000 patients with subacute stroke have been analysed. We considered as dependent variable the effectiveness of neurorehabilitation (i.e. the achieved level of independency in activities of daily living, measured using the Barthel Index, expressed in percentage of the maximum achievable improvement), and as independent variables age, time between stroke acute event and beginning of neurorehabilitation, gender, type of stroke (ischemic vs. haemorragic) and side of hemiparesis. We performed univariate analyses for computing OR with respect to different choices of threshold for dichotomizing age and time from stroke. In this analysis median value of effectiveness was used for dichotomizing subjects in good and poor responders. Then these analyses were repeated also varying the thresholdvalue of effectiveness. Finally multivariate analyses based on forward binary logistic regression were performed varying at the same time the thresholds of age and time from stroke. With respect to threshold choice, OR-values of age resulted stable, but those of time from stroke resulted more variable. Variability increased when also the threshold chosen for dichotomizing the independent variable was changed. Multivariate analyses showed that these choices could even make not statistically significant the effect of a binary prognostic factor such as gender. In conclusion, OR-values resulted affected by threshold choices. It can increase the difficulties in marking predictions of outcomes after stroke. In this study we reported a possible graphical evaluation of the variability of OR-values with respect of threshold choice, that can be helpful whenever threshold is arbitrary chosen.
\end{abstract}

Keywords: Odds ratio, logistic regression, prognostic factor, probability, stroke.

\section{INTRODUCTION}

There is a considerable body of literature concerned with the identification of factors that could affect the recovery after a neurological damage such as stroke. The probability of recovery after stroke depends on many factors, and the estimation of that probability with respect to a specific factor has been usually performed computing the odds ratio (OR). OR represents the odds that an outcome will occur given a particular exposure, compared to the odds of the outcome occurring in the absence of that exposure [1].

Given a table of contingency (Table 1), OR can easily be computed as follows [1]: OR $=\frac{a / c}{b / d}=\frac{a \cdot d}{b \cdot c}$

Table 1: Table of Contingency

\begin{tabular}{|c|c|c|c|}
\hline & \multicolumn{2}{|c|}{ Cases } \\
\hline & & $\begin{array}{c}\text { Cases } \\
\text { (good outcome) }\end{array}$ & $\begin{array}{c}\text { No cases } \\
\text { (poor outcome) }\end{array}$ \\
\hline \multirow{2}{*}{$\begin{array}{c}\text { Factor } \\
\text { under } \\
\text { analysis }\end{array}$} & Exposure & a & $\mathrm{b}$ \\
\hline & $\begin{array}{c}\text { Not } \\
\text { Exposure }\end{array}$ & c & $d$ \\
\hline
\end{tabular}

*Address correspondence to this author at the Clinical Laboratory of Experimental Neurorehabilitation, I.R.C.C.S. Fondazione Santa Lucia, via Ardeatina 306, 00179, Rome, Italy; E-mail: m.iosa@hsantalucia.it
OR is an approximation of the Relative Risk [2], the formula of which is: $R R=\frac{a /(a+c)}{b /(b+d)}$, and both are measures of association between an exposure (the independent variable) and an outcome (the dependent variable). However, OR is more widely used in many fields of biomedical research for the clear meaning of its value [1]. If the association between exposure and outcome is statistically significant, the exposure factor can be defined as a prognostic factor for stroke recovery, i.e. a characteristic, a situation or a condition of the patient that can be used to estimate the chance of recovery after stroke. The statistical significance is usually assessed using the $\chi^{2}$-test. When more than one independent factor is present, the most common analysis is the logistic regression, in particular the binary logistic regression. Regression analysis in fact provides a powerful statistical methodology for investigating relationships among independent and dependent variables [3].

A paradigmatic and well studied example is the investigation of which factors (independent variables) could predict a good recovery (dependent variable) after neurorehabilitation in subjects who had a cerebrovascular accident. Stroke is, the leading cause of disability in all industrialized countries, and the global burden of stroke is increasing worldwide [4]. For these reasons clinicians are progressively more interested in identifying which patients could be more responsive to 
specific types of rehabilitation [5-7]. This evaluation is usually performed computing the odds ratio. For example, a study conducted on 440 patients with ischemic stroke, showed that male patients had higher probability than female patients to achieve a good level of autonomy in activity of daily living (OR=3.22) [8]. Another study conducted on 270 patients showed the importance of type of stroke, reporting that patients with hemorrhagic stroke showed a probability of a high therapeutic response in terms of Barthel Index (a scale of independency in activity of daily living) with respect to patients with ischemic stroke $(\mathrm{OR}=2.48)[9]$.

For predicting a binary response from a set of binary possible predictors, it is usually performed a binary logistic regression analysis. It allows for computing an OR for each one of the factors entering into the final model.

A study conducted on 440 patients with stroke, identified the following prognostic factors of a poor motor recovery: longer time between stroke event and beginning of rehabilitation, older age, presence of aphasia, presence of unilateral spatial neglect [10]. In general, gender, age and an early onset of rehabilitation have been identified, among many other patients' features, as important prognostic factors of recovery after stroke. Prognostic factors could help into identifying low responders to neurorehabilitation and hence the patients needing more attention or those needing alternative therapeutic strategies for their augmented risk of not achieving independency in activities of daily living $[6,7]$.

Some of the above reported prognostic factors are binary variables, such as gender (male vs. female) and type of stroke (ischemic vs. haemorrhagic), but many others need to be dichotomized for computing the relevant ORs. The presence of aphasia and/or neglect could be considered as binary variables (presence or not), but their assessment usually needs the use of neuropsychological tests, and a score lower than a given threshold identifies the presence of aphasia (usually for a damage in left brain hemisphere) or neglect (usually for a damage in right brain hemisphere). Conversely, time between stroke event and beginning of neurorehabilitation as well as age of patients are two examples of continuous variables that need to be dichotomized. The choice of thresholds for this dichotomization is often an arbitrary trade-off. There are many approaches to determining thresholds, which fall into two main categories: a subjective arbitrary choice, sometimes based on previous studies (for example age $<50$ years [8]) or an objective approach in which threshold depends on data distribution (for example mean or median values [10]). Further, threshold choice could depend on the used measure unit (for example time between stroke and beginning of neurorehabilitation could be measured in days, weeks or months, and relevant threshold are commonly multiple of the used unit). But poor attention has been deserved on the effects of these thresholdchoices on the results of statistical analyses.

The aim of this study was to investigate the variability of OR-values with respect to the choice of threshold value for dichotomizing independent and/or dependent variables.

\section{MATERIAL AND METHODS}

To understand the effects of changing the threshold value for dichotomizing variables on the statistical significance of a prognostic factor, we have performed an analysis on 1000 patients with stroke in subacute phase. For the sake of simplicity, we limited our analyses to three binary factors: gender, type of stroke, side (hemisphere) of lesion. The former two factors have been known to be significant prognostic factors, conversely the last one is not expected to play a significant role on neurorehabilitation outcome [8-9]. Then, we selected two continuous variables needing to be dichotomized, both well-known as significant prognostic factors: age and interval between stroke event and beginning of neurorehabilitation [10]. According to previous studies [8-10], the achieved independency in activity of daily living at dismissal from neurorehabilitative hospital has been used for identifying the good responders to neurorehabilitation.

We performed a retrospective study on a cohort of 1000 patients with stroke in subacute phase, with the following inclusion criteria: diagnosis of first ever stroke, that was defined as a sudden, nonconvulsive, focal neurological deficit persisting for more than 24 hours [11], subacute phase of stroke (time from stroke event $<120$ days). Exclusion criteria were: subarachnoid hemorrhage, patients with previous strokes (including patients with full clinical remission), and those with other chronic disabling pathologies (ie, severe Parkinson's disease; polyneuropathy; severe cardiac, liver, or renal failure; and cancer). These criteria were similar to those of previous studies on prognostic factors [8-10].

Of many recorded data for each one of these patients, we included into the analysed database their 
age (measured in years), gender (male vs. female), the side of their lesion (right vs. left brain lesion), the time from stroke at which each patient started the neurorehabilitation (measured in days). Further, their independency in activities of daily living has been assessed using the Barthel Index (BI) [12]. The $\mathrm{BI}$ is a clinical scale that uses ten items that cover mobility and self-care domains; scores range from 0 to 100: a score of 0 indicates total dependence in ADL and a score of 100 , complete independence [12].

For normalizing data considering the initial severity of stroke effects, we have considered into our analysis the effectiveness of neurorehabilitation. Effectiveness is a commonly used parameter that reflects the proportion of potential improvement that was achieved at discharge from rehabilitation hospital. It has been calculated as follows $[12,13]$ :

$$
\text { Effectiveness }=\frac{\mathrm{BI}_{\text {discharge }}-\mathrm{BI}_{\text {admission }}}{\mathrm{BI}_{\text {max }}-\mathrm{BI}_{\text {admission }}} \cdot 100
$$

with $\mathrm{BI}_{\text {discharge }}$ and $\mathrm{Bl}_{\text {admission }}$ being the $\mathrm{Bl}$-scores at discharge and admission, respectively, and $\mathrm{BI}_{\max }$ the maximum of BI-scale, i.e., 100: if a patient achieves this highest possible score of Barthel Index after rehabilitation, the effectiveness is $100 \%$.

Firstly, for the sake of simplicity, we performed univariate analyses separately on age and time from stroke. The threshold chosen for dichotomizing these two variables was changed for age from 40 to 85 years with step 1 year, and for time from 1 to 45 days with step 1 day. We computed the odds ratio (OR) of achieving a good independency in activities of daily living. Mean, standard deviation and coefficient of variation $(C V=$ standard deviation / mean * 100) were computed for all the OR-values at varying of threshold(s).

$\chi^{2}$ test was performed for assessing the significance of $\mathrm{OR}$ in univariate analysis. $\chi^{2}$ was computed as follows:

$$
\chi^{2}=\frac{(a \cdot d-b \cdot c)^{2}-(a+b+c+d)}{(a+b) \cdot(c+d) \cdot(a+c) \cdot(b+d)}
$$

Beyond the statistical significance of $\chi^{2}$-test, according to Cohen [14] the effect of the factor is considered not trivial for $\mathrm{OR}>1.5$. In particular the effect was considered small for an OR between 1.5 and 3.5, moderate between 3.5 and 9 , and large if $O R>9$.
Then, to assess also the effect of threshold choice on the dependent variable, other univariate analyses were performed modifying this threshold from $31 \%$ to $76 \%$ of effectiveness with step $1 \%$. A number of 2116 $(46 * 46)$ computations have been performed for age and 2116 performed for time in that analysis using MATLAB $®$.

Finally, we performed multivariate analyses using forward binary logistic regression with the same dependent variable above reported (effectiveness, using median value as threshold), and with the following independent variables: age (from 40 to 85 with step 5 years), time from stroke (from 5 to 50 with step 5 days), gender (male vs. female). When the factor entered into the model the OR resulted equal to the exponential function of the regression coefficient, otherwise OR was fixed at 1 for representing its not significant role. A total of 100 multivariate regression analyses have been performed in that analysis using the software SPSS 17.0. This software provides together with $p$-values also the regression coefficient $\beta$ for each factor: it is the estimated increase in the log odds of the outcome per unit increase in the value of the exposure, implying that its exponential function of $\exp (\beta)$ coincides with the OR associated with a oneunit increase in the exposure factor [1].

\section{RESULTS}

\section{Sample Description}

The data of 1000 patients in subacute phase of stroke (time from stroke event $<120$ days) have been analysed: 557 males vs. 443 females; 550 with damage in left hemisphere and 450 in right hemisphere; 841 with ischemic and 159 with haemorrhagic stroke. Their mean age was $67 \pm 14$ years, with a mean time from stroke at the admission in rehabilitation hospital of $25 \pm 24$ days. The mean score of Barthel Index was $31.16 \pm 27.48$ at the admission and $66.26 \pm 31.03$ at dismissal. The mean effectiveness was $58.33 \pm 33.21 \%$ with a median value of $58.38 \%$. This median value was used to dichotomize patients between poor (effectiveness < 58.38\%) and good (>= $58.38 \%$ ) responders to neurorehabilitation. The distributions of effectiveness with respect of age and time from stroke are shown in Figure 1.

\section{Univariate Analysis}

Figure 2 reports the values of OR (and of $R R$ ) associated to a good outcome after neurorehabilitation 

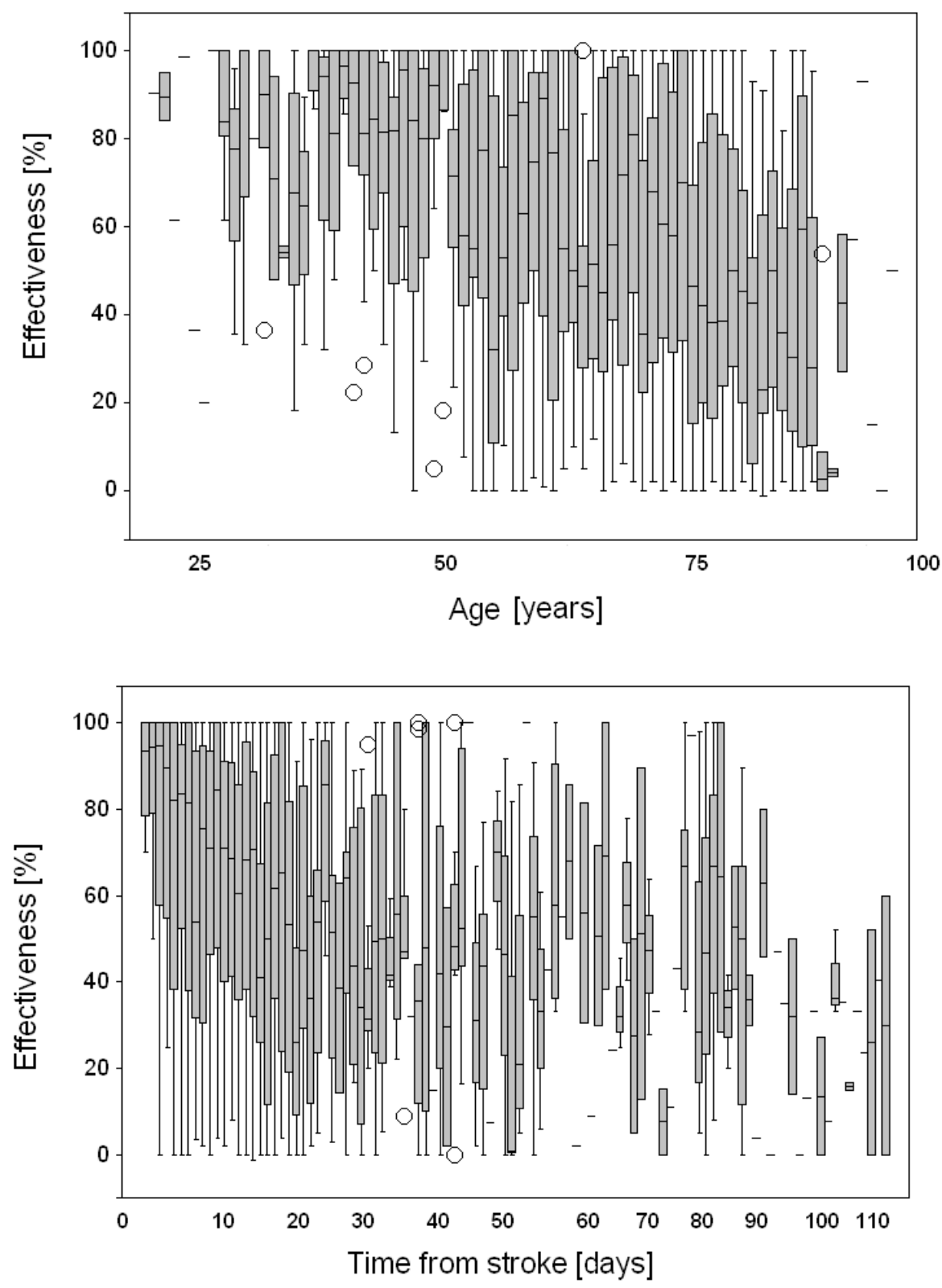

Figure 1: Box-plot of distribution of effectiveness of rehabilitation on recovery of activities of daily living with respect to age of patients (above) and time between stroke event and beginning of rehabilitation (below). The boxes show the first quartile, median, and third quartile values, the whiskers represent the most extreme values within 1.5 times the interquartile range from the ends of the box, the circles represent the outliers (data with values beyond the ends of the whiskers).

(i.e., to subjects in the third and fourth quartiles of effectiveness) for the 46 possible values of the thresholds chosen for age (above on the left) and for time from stroke (above on the right). The mean OR was $2.97 \pm 0.75(\mathrm{CV}=25.4 \%)$ for age and $3.60 \pm 2.95$ $(\mathrm{CV}=82.0 \%)$ for time.

All the values of OR resulted statistically significant $(p<0.05)$. Furthermore, OR was higher than 1.5 for all the possible threshold choices. Hence both these factors resulted playing a not trivial effect in the whole ranges of threshold-values, in accordance with their prognostic role reported in literature. However, the effect of age was moderate if the threshold was chosen in the range between 41 and 54 years and small out of this range, with a negative peak of $\mathrm{OR}=1.86$ at 69 years.

For time, the range of values covered by $\mathrm{OR}$ is even wider, with a large effect for a time of 2 days (but with a $p=0.045$ close to the critical alpha level) or 3 days $(p=0.002)$, moderate for $4-5$ days and small for a threshold chosen after 5 days $(p<0.01$ for all these choices). This variability is reflected by the high $\mathrm{CV}=82.0 \%$ of OR-values. The middle plots of Figure 2 represents the values of $a, b, c$ and $d$ with respect to 

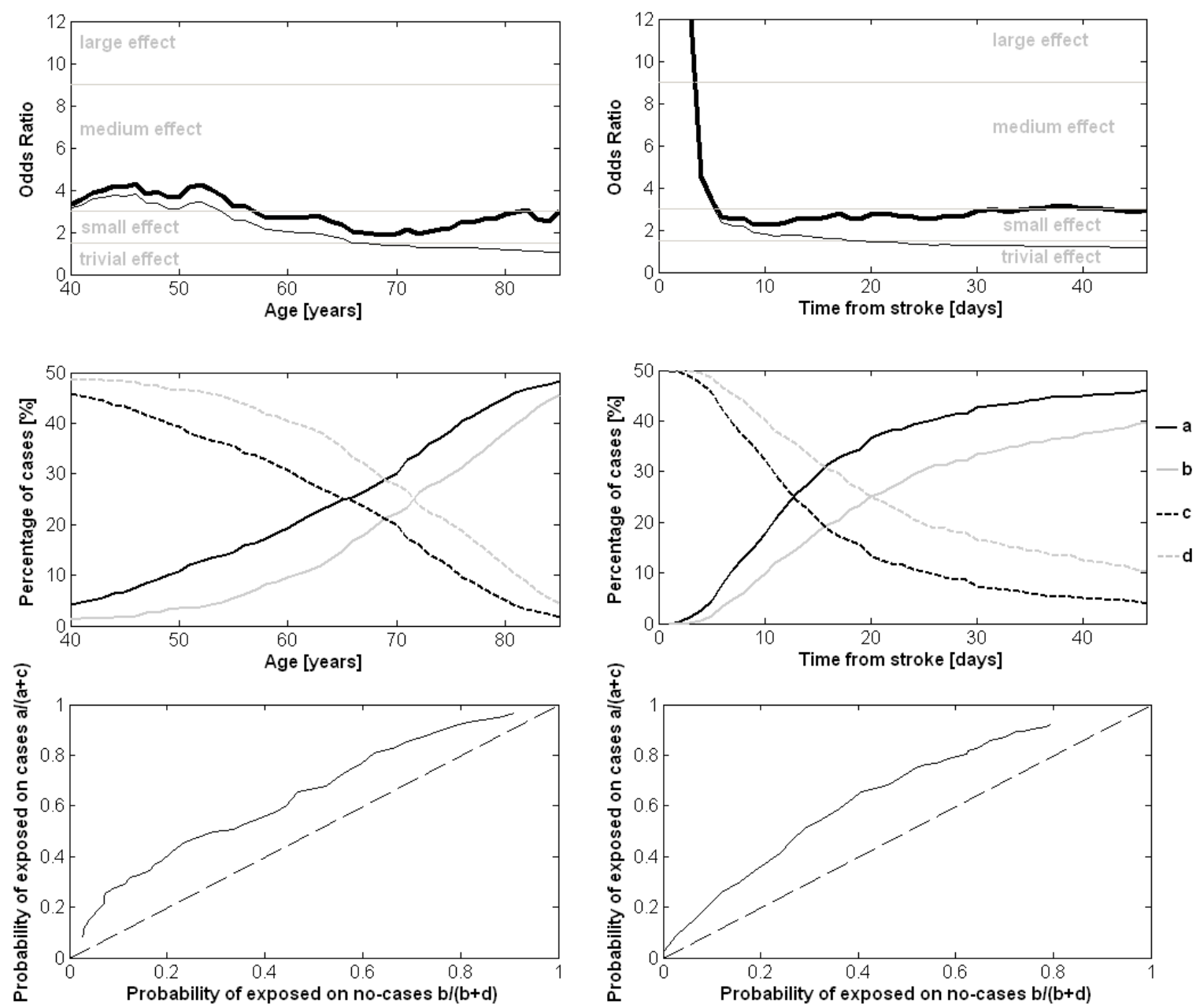

Figure 2: Above: values of OR (bold lines) and RR (thin lines) for age (on the left) and for time from stroke (on the right) at varying the threshold-values for dichotomizing the independent variables age (on the left) and time from stroke (on the right). In the middle: the values of $a, b, c, d$ at varying of thresholds. Below: probability of exposed subjects on cases plotted in respect of exposed subjects on no-cases.

threshold choice. The functions $a(x)$ and $b(x)$ are cumulative distribution functions, whereas $c(x)$ and $d(x)$ are their complementary functions, respectively. As well known [2], the OR approximates the RR only when $a<<c$ and $b<<d$, but as shown in Figure 2 it can depend on threshold choice. For the RR is possible to depict a trend analogous to that depicted in Receiver Operating Characteristic curve (lower plots of Figure 2) when the probability of exposed on cases $(a /(a+c))$ is plotted vs. the probability of exposed in no cases $(b /(b+d))$. However, an analogous plot for OR was not possible because $a / c$ and $b / d$ do not have the limit of 1 .

Figure 3 shows the odds ratio for age (left) and time from stroke (right) when not only the threshold for dichotomizing the exposure factor was varied, but also the threshold for defining the subject as a case (good responder to neurorehabilitation) or not (poor responder) was changed (i.e. varying the threshold for dichotomizing the dependent variable effectiveness). For age (left plot), the mean OR was $3.00 \pm 0.94$ $(C V=31.35 \%)$, and all the OR values resulted statistically significant $(p<0.05)$. For the for time from stroke (right plot), the mean OR was $2.70 \pm 1.17$ $(C V=43.47 \%)$, and the $\chi^{2}$-test showed not statistically significant effect when threshold was 2 days and threshold for effectiveness was lower than 58\% ( $p$ value in the range between 0.0504 and 0.2498 ) or higher than $71 \%$ ( $p$-value in the range between 0.1391 and 0.1745 ).

In that Figure 3, the two bold black lines correspond to those reported in Figure 2 for an effectivenessthreshold equal to the median of effectiveness $(58.38 \%)$. The choice of that threshold lied in the area of high OR values for time, but not for age. Higher ORvalues for age were in fact related to lower values of effectiveness-threshold. 

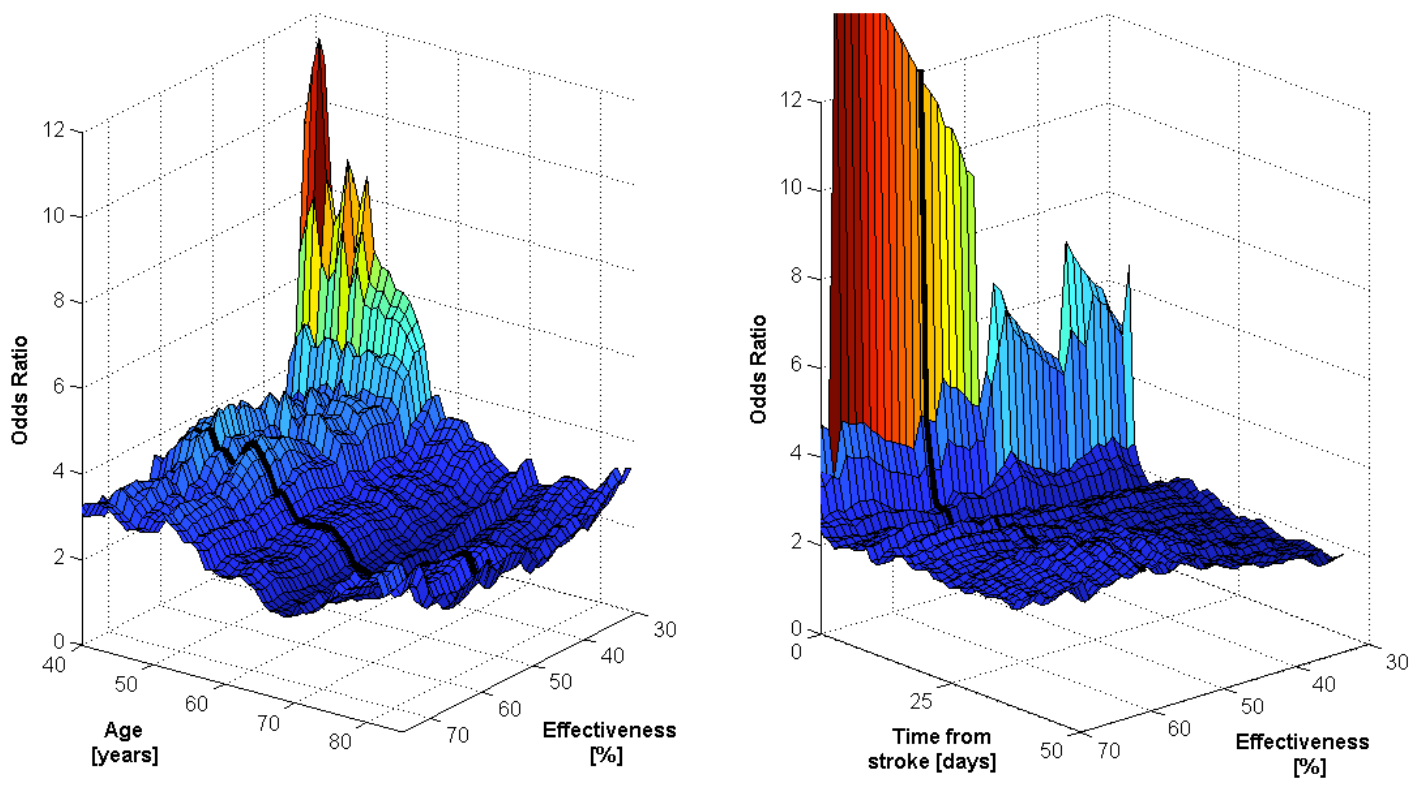

Figure 3: Odds Ratio of age (on the left) and time from stroke (on the right) for variations in the threshold of independent (age on the left and time on the right) and dependent (effectiveness) variables. The bold black lines correspond to those reported in Figure 2 for an effectiveness of $58.38 \%$.

\section{Multivariate Analysis}

When binary logistic forward regressions have been applied for identifying the patients with an effectiveness of treatment in the higher two quartiles, neither side of lesion nor type of stroke resulted playing a statistically significant role. We analysed which one of the following three factors: age, time from stroke, and gender a significant role entering into the model of a binary logistic regression for predicting a good motor recovery defined by an effectiveness higher than $58-38 \%$ (median value of effectiveness). Figure 4 reports the OR computed as the $\exp (\beta)$ entered into that model at varying of threshold for time and age.

The OR related to age did not played a statistically significant role in about half of the performed regressions ( $p>0.05$ in 49 on 100 comparisons) corresponding at the age ranges $40-45$ and $60-65$ years (a range not far from the negative peak observed
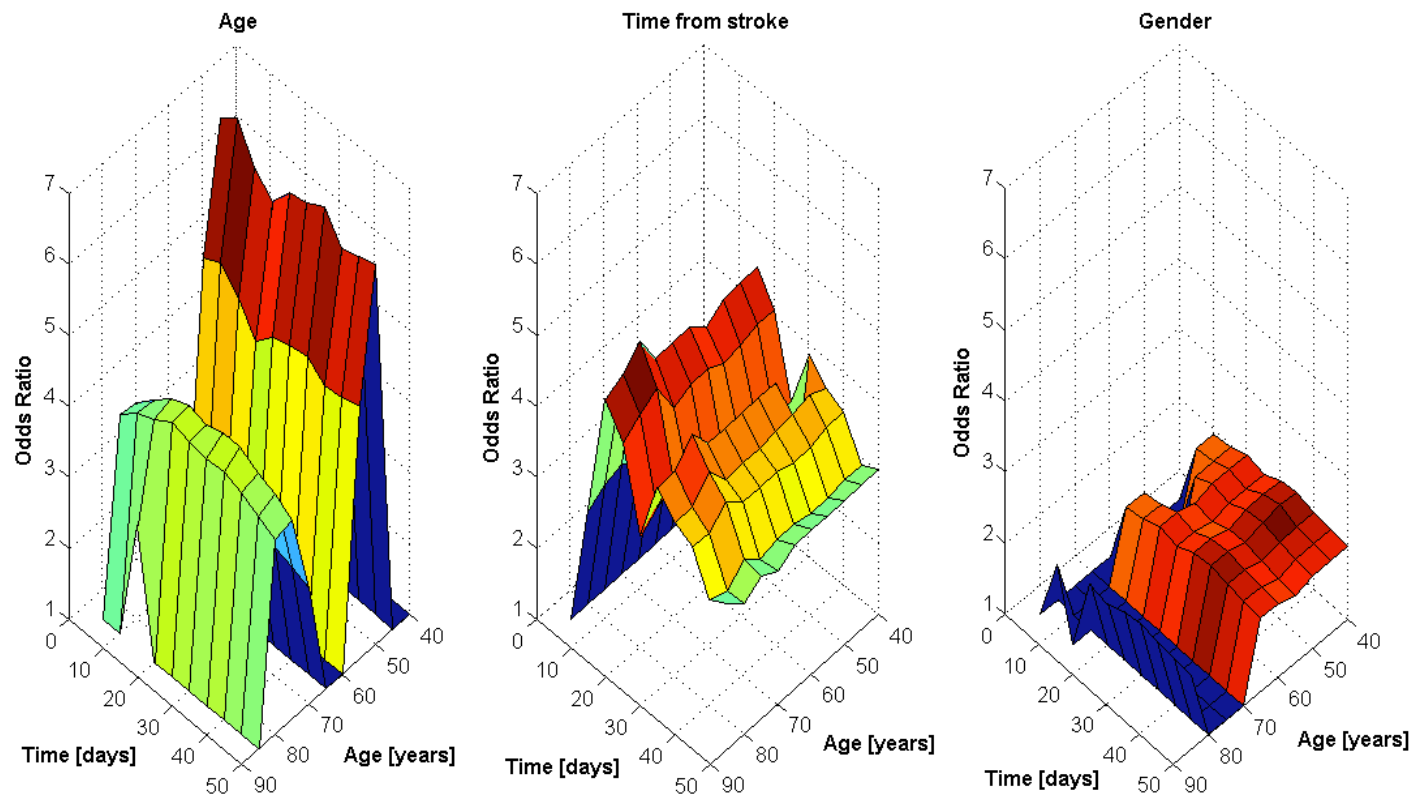

Figure 4: OR-values for age (on the left), time from stroke (in the middle), and gender (on the right) related to the variations in the threshold-values of time from stroke and age. 
in univariate analysis that was at 69 years). It should be noted that in these ranges age did not entered into the model.

The OR related to time $>5$ days were in mean $3.45 \pm 0.60(\mathrm{CV}=17.34 \%)$. For time lower than 5 days, differently from univariate analysis result, time from stroke was not a significant factor (in Figure 4, OR was graphically reported as 1 when the factor did not entered into the model).

The case of gender was particularly interesting because this factor is binary, but our results showed that its role can be affected by the threshold choices related to other factors, and it mainly entered into the model if the age-threshold was lower than 70 and the time-threshold was longer than 20 days (for these choices OR=1.98 $\pm 0.25, \mathrm{CV}=12.59 \%$ ).

\section{DISCUSSION}

Despite the difficulties in predicting the possibilities of recovery in subjects with stroke for the high variability of the effects of this pathology, younger age, short time between stroke event and beginning of neurorehabilitation, as well as male gender have been demonstrated to be factors associated to a higher probability of a good recovery of independence in activity of daily living [8-10]. These factors resulted for most of the performed analyses as playing a significant role, but not for all of them.

Univariate analysis showed that age and time from stroke played a statistically significant role for all the chosen thresholds. Despite it, their OR-values were widely variable, ranging from a high to a small effect. Further, when also the threshold of effectiveness was varied, particular combinations of thresholds make the role played by time from stroke not statistically significant.

When multivariate analyses have been performed, not only time, but even age resulted a not statistically significant predictor of good recovery under particular threshold choice related conditions. The most astonishing result was that even the role played by gender, that is a binary factor without any need of threshold choice, can be significant or not in relationship to the threshold choices performed on the other factors in multivariate analysis.

By way of example, the fact that the high time-effect for short time resulted not statistically significant in the multivariate analysis could be due to the fact that a patient is able to start the neurorehabilitation within few days from the event only if the severity of stroke was weak and he/she is not an elderly. So, in multivariate analysis the effect of this factor lose its significance because younger age was a best predictor of a good motor recovery. It could also explain the increased ORvalues for age in subjects between 45 and 50 years old. Another reason could be the reduced size of subsample of subjects with a time lower than 5 days that could have reduced the significant of logistic regression results $(5.9 \%$, such as for age of 40 or less years: $5.4 \%$ ) generating an artefact effect. Some of our results could be due to casual artefacts related to the many performed comparisons. However this possibility is quite limited by the width of sample, that in turn could have increased the risk of error type I. Further, it should be considered that when extreme values were chosen for thresholds, the sub-sample resulted formed by few subjects, increasing the risk of artefact affecting the statistical significance of results.

Despite the specific differences, we found maximum values of OR for time in the range 15-25 days, independently by age-threshold, in accordance with what reported in literature about higher probability of good recovery if treatment begins within 20 days after the acute event [16].

Another interesting aspect of our results is a "lack" of statistical significance of the factor age in the range 60-65 years (or 65-70 for univariate analysis). It could be due to the fact that age plays a fundamental role when the threshold was chosen at its extremities, differentiating between young adults and others or between old elderly and others. Further, we have considered a logistic regression model including a constant, and when age-threshold was between 60 and 65 years, the inclusion of age into the model did not significantly improve the variance already explained by that constant. It was probably due to the fact that the median effectiveness $(58.68 \%)$ was close to the mean value of that subgroup of subjects $(62.84 \%)$. This hypothesis was supported by the fact that if the multivariate logistic regression was repeated without including a constant into the model, the role of age became statistically significant, and even with a high effect (for example for threshold values of 60 years and 20 days, the OR of age was 14.64, $p<0.001$ ). This result was particularly important because the age of 65 years is often chosen as threshold because the World Health Organization used it for defining "elderly".

According to literature we found that male gender had more possibilities of a good recovery than women 
$[8,17]$, but our results showed that it was true only when subjects are younger than 70 years old, probably for the preserved muscle strength in not elderly men [18].

Finally, type of stroke (ischemic vs. haemorragic) did not enter into the model in our study. It could be due to the high difference in sample size between ischemic stroke $(84.1 \%)$ vs. haemorragic stroke (15.9\%). A previous study reported an OR $=2.48$ for achieving a good motor recovery in haemorragic stroke in respect of ischemic stroke, but that study was performed comparing two samples having the same size [9].

In conclusion, being the aim of researchers to identify the OR-values independently by their arbitrary choices, the variability of the values of OR and related statistical level of $p$ should be assessed. Here we have shown a simple approach to graphically evaluate this variability with respect to threshold choices.

\section{REFERENCES}

[1] Szumilas M. Explaining Odds Ratios. J Can Acad Child Adolesc Psychiatry 2010; 19: 227-9.

[2] Armitage P, Berry G, Matthews JNS. Statistical methods in epidemiology in Statistical Methods in Medical Research, Wiley-Blackwell, 4th ed., 2001; pp. 648-716.

[3] Greenhouse JB, Bromberg JA, Fromm D. An introduction to logistic regression with an application to the analysis of language recovery following a stroke. J Commun Disord 1995; 28: 229-46. http://dx.doi.org/10.1016/0021-9924(94)00011-N

[4] Donnan GA, Fisher M, Macleod M, Davis SM. Stroke. Lancet 2008; 371: 1612-23. http://dx.doi.org/10.1016/S0140-6736(08)60694-7

[5] Dobkin BH, Nadeau SE, Behrman AL, Wu SS, Rose DK, Bowden M, Studenski S, Lu X, Duncan PW. Prediction of responders for outcome measures of locomotor. Experience Applied Post Stroke trial. J Rehabil Res Dev 2014; 51: 39-50. http://dx.doi.org/10.1682/JRRD.2013.04.0080

[6] Morone G, Bragoni M, losa M, et al. Who may benefit from robotic-assisted gait training? A randomized clinical trial in patients with subacute stroke. Neurorehabil Neural Repair 2011; 25: 636-44.

http://dx.doi.org/10.1177/1545968311401034

[7] Morone G, losa M, Bragoni M, et al. Who may have durable benefit from robotic gait training? a 2-year follow-up randomized controlled trial in patients with subacute stroke. Stroke 2012; 43: 1140-2.

http://dx.doi.org/10.1161/STROKEAHA.111.638148

[8] Paolucci $S$, Bragoni $M$, Coiro $P$, et al. Is sex a prognostic factor in stroke rehabilitation? A matched comparison. Stroke 2006; 37: 2989-94.

http://dx.doi.org/10.1161/01.STR.0000248456.41647.3d

[9] Paolucci S, Antonucci G, Grasso MG, et al. Functional outcome of ischemic and hemorrhagic stroke patients after inpatient rehabilitation: a matched comparison. Stroke 2003; 34: 2861-5.

http://dx.doi.org/10.1161/01.STR.0000102902.39759.D3

[10] Paolucci S, Antonucci G, Pratesi L, et al. Functional outcome in stroke inpatient rehabilitation: predicting no, low and high response patients. Cerebrovasc Dis 1998; 8: 228-34. http://dx.doi.org/10.1159/000015856

[11] Foulkes MA, Wolf PA, Price TR, Mohr JP, Hier DB. The stroke data bank: design, methods, and baseline characteristics. Stroke 1988; 19: 547-54.

http://dx.doi.org/10.1161/01.STR. 19.5.547

[12] Mahoney FI, Barthel DW. Functional evaluation: the Barthel index. Md State Med J 1965; 14: 61-5.

[13] Shah S, Vanclay F, Cooper B. Efficiency, effectiveness, and duration of stroke rehabilitation. Stroke 1990; 21: 241-6. http://dx.doi.org/10.1161/01.STR.21.2.241

[14] Vanclay F. Functional outcome measures in stroke rehabilitation. Stroke 1991; 22: 105-8.

http://dx.doi.org/10.1161/01.STR.22.1.105

[15] Cohen J. Statistical power analysis for the behavioral sciences 2nd ed. Erlbaumm 1998.

[16] Rossi PW, Forer S, Wiechers D. Effective rehabilitation for patients with stroke: analysis of entry, functional gain, and discharge to community. J Neurol Rehabil 1997; 11: 27-33.

[17] Glader EL, Stegmayr B, Norrving B, et al. Sex differences in management and outcome after stroke: a Swedish national perspective. Stroke 2003; 34: 1970-5.

http://dx.doi.org/10.1161/01.STR.0000083534.81284.C5

[18] Sinaki M, Nwaogwugwu NC, Phillips BE, Mokri MP. Effect of gender, age, and anthropometry on axial and appendicular muscle strength. Am J Phys Med Rehabil 2001; 80: 330-338. http://dx.doi.org/10.1097/00002060-200105000-00002

[19] Matthews R. Storks deliver babies $(p=0.008)$. Teaching Statistics 2000; 22: 36-8.

http://dx.doi.org/10.1111/1467-9639.00013

http://dx.doi.org/10.6000/1929-6029.2014.03.04.3

(c) 2014 losa et al.; Licensee Lifescience Global.

This is an open access article licensed under the terms of the Creative Commons Attribution Non-Commercial License (http://creativecommons.org/licenses/by-nc/3.0/) which permits unrestricted, non-commercial use, distribution and reproduction in any medium, provided the work is properly cited. 